\title{
FAST STEREO MATCHING METHOD USING EDGE TRACTION
}

\author{
Zheng-dong Liu, Ying-nan Zhao and Jing-yu Yang \\ Department of Computer Science, Nanjing University of Science and Technology, Nanjing \\ 210094, P.R. China
}

Abstract: Combining the reliable edge feature points and area similarity, the fast stereo matching algorithm using edge traction was presented. First, find valid disparity set of feature points and traverse combinations of adjacent points' disparities, obtain the valid disparity set of featureless points using dynamic program, then, generate the initial sparse disparity space using area similarity. The algorithm reduces the computation complexity of disparity space and decreases the possibility of mismatching illusion. Under the uniqueness constraint, integral dense disparity map and occlusion area can be obtained by collision detection. Experiment on real visual images is performed to verify the feasibility and effectiveness of this algorithm.

Key words: Stereo Matching, Edge Feature, Disparity, Occlusion Detection

\section{INTRODUCTION}

The stereo matching is used for obtaining reliable, dense and smooth disparity maps. The area-based methods can produce dense disparity map ${ }^{[1][2]}$, but the match is very sensitive to noise and light reflection. The featurebased methods can obtain highly reliable match of the feature points ${ }^{[3][4]}$, but dense disparity map is available only by interpolation.

For the research of moveable machine with visual system, real-time is more required then high precision. In this paper, we present a fast stereo algorithm using edge traction. Combining area-based and feature-based methods can produce more precise disparity map. Relatively reliable edge is used to restrict the calculation of disparity scope of featureless points, which 
also helps to decreases the possibility of mismatch illusion when detect occlusion area. To demonstrate the effectiveness of the algorithm, we provide experimental data from a real image pair. Conclusion is drawn at last.

\section{FAST STEREO MATCHING USING EDGE TRACTION}

Edge feature points are relatively less sensitive to noise. We use the LoG method to get the feature image $F^{\prime}(x, y)$. Construct a feature point label set:

$$
J=\left\{j_{1}, j_{2}, \ldots, j_{n}\right\}
$$

The $n$ is the number of feature points, $j_{i}$ is the valid disparity set of feature point $i$. Disparities outside the label set are considered as forbidden area. The valid disparity set of featureless point can be obtained by the assortment of disparities of the adjacent feature points using dynamic program. Assume $\left(x_{a}, y\right) \in F^{\prime}$ and $\left(x_{b}, y\right) \in F^{\prime}$ are adjacent feature points. Define left compatible sequence $L$ and right compatible sequence $R$ :

$$
\begin{aligned}
& L=\left\{l_{i} \mid x_{a}<l_{i}<x_{b}, 0<i \leq M\right\} \\
& R=\left\{r_{j} \mid x_{a}+d_{a}<r_{j}<x_{b}+d_{b}, d_{a} \in j_{a}, d_{b} \in j_{b}, 0<j \leq N\right\}
\end{aligned}
$$

We use dynamic compatibility to match two sequences. Let $H(L, R)$ represents the total minimal accumulated distortion distances. For $l_{i} \in L, r_{j} \in R$, their distortion distance $h\left(l_{i}, r_{j}\right)$ is absolute value of difference. According to the continuity constraint, the result of compatibility matching is monotonic increasing. If $H\left(l_{i}, r_{j}\right)$ is the minimal accumulated distortion distance at $\left(l_{i}, r_{j}\right)$, the new distance value can be defined as:

$$
\begin{aligned}
& H\left(l^{\prime}, r^{\prime}\right)=\min \left\{H\left(l_{i}, r_{j}\right)+h\left(l_{i+1}, r_{j}\right), H\left(l_{i}, r_{j}\right)+2 h\left(l_{i+1}, r_{j+1}\right),\right. \\
& \left.H\left(l_{i}, r_{j}\right)+h\left(l_{i}, r_{j+1}\right)\right\}
\end{aligned}
$$

The final minimal accumulated distortion distance is:

$$
H(L, R)=\frac{1}{M+N} H\left(l^{\prime}, r^{\prime}\right)
$$

Traveling through all the assortments of $j_{a}$ and $j_{b}$, we can find out the valid disparity set of each featureless point. Area outside the valid disparity 
set is also defined as forbidden area. Based on the valid disparity set of each point, the disparity space with sparse match values can be constructed. Most methods traverse all disparities and pixels to construct the disparity space ${ }^{[2][5]}$. For $M \times N$ gray image, $W \times W$ correlative window and $D$ maximal disparity, their computation complexity is $O\left(M \times N \times W^{2} \times D\right)$, a computation complexity of this algorithm was reduced to $O\left(M \times N \times D^{2}\right)$.

Define the left line of sight $L S$ and corresponding right line of sight $R S$ at pixel $\left(x_{i}, y_{i}\right)$ as a point set in the disparity space:

$$
\begin{aligned}
& L S=\left\{(x, y, d) \mid x=x_{i}, y=y_{i}, 0<d<d_{\text {max }}\right\} \\
& R S=\left\{(x, y, d) \mid x=x_{i}+\left(d-d_{i}\right), y=y_{i}, 0<d<d_{\text {max }}\right\}
\end{aligned}
$$

In General, points in occlusion area have smaller match values along their line of sight. The point with the highest match value, if had conflict on its left line of sight, is marked as unmatchable point. When all the points which have a larger match values than threshold are marked as unmatchable points, they can be determined as occlusion area. Otherwise, disparity with the highest match value could be the final matching disparity. In many instances, there are some feature points between occluded areas, it restricts valid disparity scope of featureless points and avoid mismatching illusion when search incorrect area with high match values.

\section{EXPERIMENTAL RESULTS}

We have applied the algorithm to the corridor images from Carnegie Mellon University. Figure 1(a), (b), and (e) show the image pair and its ground-truth disparity map. Figure 1(c) shows the image of feature image by LoG Filter with $\sigma=0.5$. Disparity map of edge feature points was shown in 1(d). Figure 1(f) shows the disparity map as a result. Black areas in the disparity map are detected occlusions. Running time was only $3.2 \mathrm{~s}$ for decreasing valid disparity scope and processing without iteration. Table 1 shows the statistics data of the experiment.

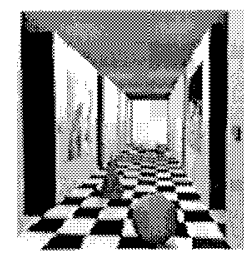

(a)

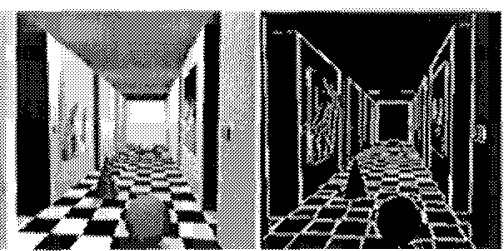

(c) 


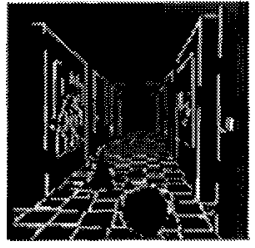

(d)

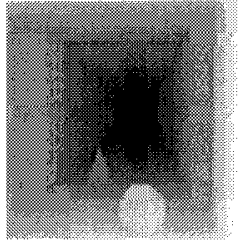

(e)

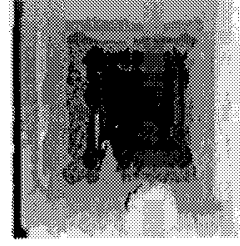

(f)

Figure 1. Experimental Results. (a) Left image, (b) right image, (c) left edge features image, (d) feature disparity map, (e) ground true disparity map, (f) disparity map as a result.

Table 1. Statistics data of the experimental result

\begin{tabular}{llll}
\hline & Pixel Found & Correct Match & \%Correct Rate \\
\hline Available Feature & 7817 & 7766 & 99.35 \\
Featureless & 54558 & 47329 & 86.75 \\
Occluded Points & 3161 & 1020 & 32.27 \\
Total & 65536 & 56115 & 85.62 \\
\hline
\end{tabular}

\section{CONCLUSION}

In the algorithm, the edge feature point restricted valid disparity set of the featureless points under the uniqueness and continuity constraint. There are the following advantages. First, Computational complexity and searching area was reduced. Efficiency of matching was improved for request of realtime processing. At last, searching along the lines of sight and threshold of match value can determine occlusion and avoid illusion in a certain extent.

\section{REFERENCES}

1. T. Kanade, M. Okutomi. A stereo matching algorithm with an adaptive window: Theory and experiment. IEEE Trans. on Pattern Analysis and Machine Intelligence, 1994, 16(9): 920 932.

2. C. L. Zitnick, T. Kanade. A Cooperative Algorithm for Stereo Matching and occlusion Detection. IEEE Trans. on Pattern Analysis and Machine Intelligence, 2000, 22(7): 675 684.

3. H. Baker, T. Binford. Depth from Edge and Intensity Based Stereo. In: Proceedings of the 7th International Joint Conference on Artificial Intelligence, Vancouver, 1981. 631 636.

4. W. Grimson. Computational experiments with a feature based stereo algorithm. IEEE Trans. on Pattern Analysis and Machine Intelligence, 1985, 7(1): 17 34.

5. Qiuming Luo, Jingli Zhou. Stereo matching and occlusion detection with integrity and illusion sensitivity. Pattern Recognition Letters, 2003, 24(9-10): 1143 1149. 